\title{
Physical modelling of the motion of a liquid metal melt along the surface of a heated rod
}

\author{
Pavel D. Lobanov ${ }^{1,2 *}$, Eduard V. Usov ${ }^{1}$, Ilya A. Klimonov ${ }^{1}$, Alexander E. Kutlimetov ${ }^{1}$, \\ Anton A. Butov ${ }^{1}$, Vladimir I. Chukhno ${ }^{1}$, Ivan G. Kudashov ${ }^{1}$, Alexander I. Svetonosov ${ }^{1,2}$, \\ and Nikolay A. Pribaturin ${ }^{1,2}$ \\ ${ }^{1}$ Nuclear Safety Institute of the Russian Academy of Sciences, Novosibirsk Branch, Novosibirsk, \\ 630090, Ak. Lavrentiev Ave., 1, Russia \\ ${ }^{2}$ Kutateladze Institute of Thermophysics SB RAS, Novosibirsk, 630090, Ak. Lavrentiev Ave., 1, \\ Russia
}

\begin{abstract}
The investigation of the melt flow of a liquid metal along the surface of a heated rod is carried up. In the experiments, the metal from the upper volume was drained over the surface of a quartz tube, heated from the inside. This pipe is used to simulate a fuel column. A high-speed video of the process was produced. Data are obtained on the features of the flow of a metal melt. The stages of formation of droplets are shown. The data on the shape and velocity of the droplet movement are given. It is shown that in the air atmosphere around the test section when the first drop passes on the surface of quartz, a trace is formed, along which the metal moves in the future. Direct and curvilinear rivulet flows on the rod's surface are also observed.
\end{abstract}

\section{Introduction}

One of the most serious accidents in the operation of nuclear power plants is the melting of the claddings of fuel elements with the subsequent release of radioactive substances into the environment. To predict the behavior of the cladding material in an emergency situation, a numerical prediction based on the experimental data is required.

Full-scale experiments of the destruction and melting of the fuel pin's cladding are extremely difficult and very expensive. In addition, in such conditions it is extremely difficult to obtain data about the process in real time. High temperature leads to the failure of various measuring sensors and thermocouples. However, the information necessary for verification and validation of numerical codes can be obtained from relatively simple experiments. Therefore, experiments are carried out recently using low-melting metals and model substrates [1-3]. Recently, papers have appeared in which low-melting metals are used to simulate the interaction of the melt of a reactor core with a pool filled with water. The purpose of the present paper is a physical modeling of the motion of a liquid metal melt along the surface of a heated rod. The lead bismuth eutectic alloy melt was used as the model of melting fuel cladding.

* Corresponding author: lobanov@itp.nsc.ru 


\section{Experimental setup}

This paper is devoted to the investigation of the motion of a model liquid-metal melt over a surface imitating a fuel column. The eutectic lead-bismuth alloy freely flowing along a cylindrical surface made of quartz was chosen as the model material of the shell. In order to perform the measurements, high-speed video was used. During the experiments, the initial temperatures of the melt and the surface of the model varied. In this paper, the experiments were carried out in an air atmosphere.

The setup was a vertical quartz tube $l$ with an external diameter of $15 \mathrm{~mm}$ (see Figure 1). This tube was used to simulate the fuel column, because it is known [4] that the contact angle of wetting for lead on a quartz substrate is approximately the same as that for stainless steel and uranium dioxide. A quartz rod 2 was installed in the inner cavity of the tube, on which the nichrome wire 3 was wound. The wire was connected to a regulated laboratory current source LATR with a maximum power of $2000 \mathrm{~W}$. During the experiments, the voltage was $50 \mathrm{~V}$, the current was $0.5 \mathrm{~A}$. This allowed the outer surface of the tube to be heated to a temperature of $175^{\circ} \mathrm{C}$. The surface temperature of the tube was monitored with a Fluke Ti32 thermal imager. The experimental setup is installed in the chemical cabinet.

To form a metal melt flow on the surface of the quartz tube, the upper container 4 initially filled with a model metal 5 (a lead-bismuth eutectic alloy in the solid state) was installed in its upper part. During the experiments, the quartz rod was heated by means of an electric current. The model metal was additionally heated with hot air. As a result, the metal was melted and its melt penetrated a narrow gap between the surface of the tube and the lower part of the container. The gap width was $0.5 \mathrm{~mm}$.

Further, the video was recorded for the process of melt flow and further processing on a computer.

\section{Experimental results}

In this paper preliminary experimental results are presented. In order to analyze the behavior of the melt of liquid metal on the not wetting surface of the quartz pipe the experiments were carried out in the air atmosphere around the test section. The overheating of the upper container in which the model metal in solid state was positioned in the beginning of our experiments was relatively small. This allows us to perform the investigation of the flow with very low flow rates of liquid metal, such as formation and movement of single drops and, for higher flow rates, rivulet flow.

The sequence of frames during the formation of a drop of liquid metal on the surface is presented on the figures 2 and 3. The side view of the drop formation process is presented on the figure 2. The front view is presented on the figure 3. The time step between frames in these experiments is $1 / 3 \mathrm{sec}$. During the time of the formation of the drop its volume is grows and in some moment the critical volume (and mass) is achieved and droplet start to move. It was found that the average velocity of droplets at the distance $30 \mathrm{~mm}$ from the release point is about $0.26 \mathrm{~m} / \mathrm{s}$.

After the droplet release the wake can occur (see last frames on the figure 3 ). In the future, the metal prefer to move in the wake of the first droplet. For different test cases the shape of the wake can be either direct or curvilinear (see figure 4). Thus rivulet flow is formed. We can observe the process of movement of liquid metal in the rivulet on the surface of the rod. But the estimation of movement characteristics is impossible. However we can measure the flow rate in the rivulet when observe the droplets formation on the rivulet's end at the bottom part of the experimental model. 


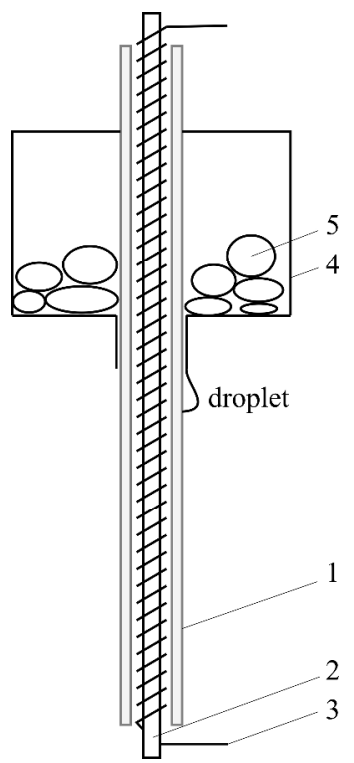

Fig. 1. The scheme of the experimental setup.
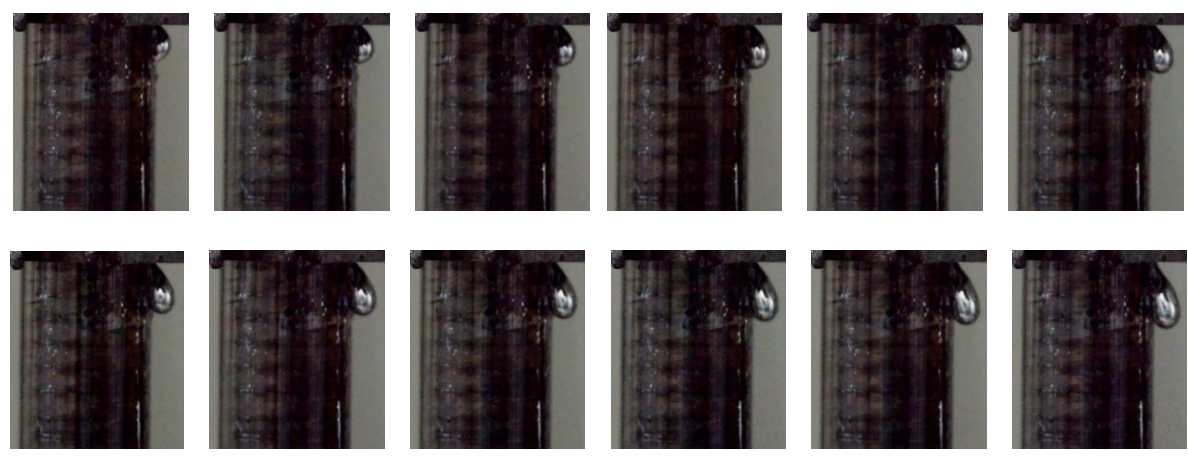

Fig. 2. Droplet formation stages. Side view.
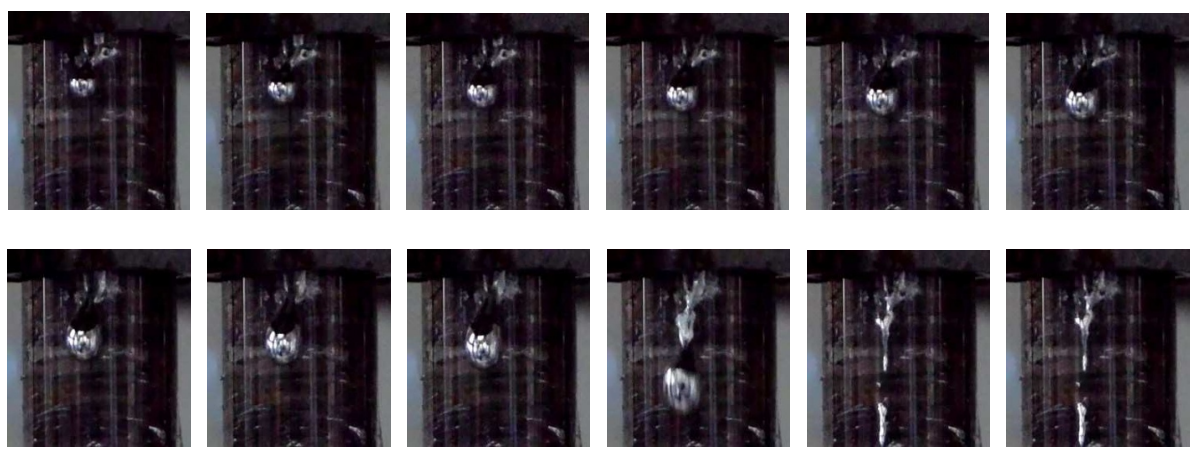

Fig. 3. Droplet formation stages. Front view. 

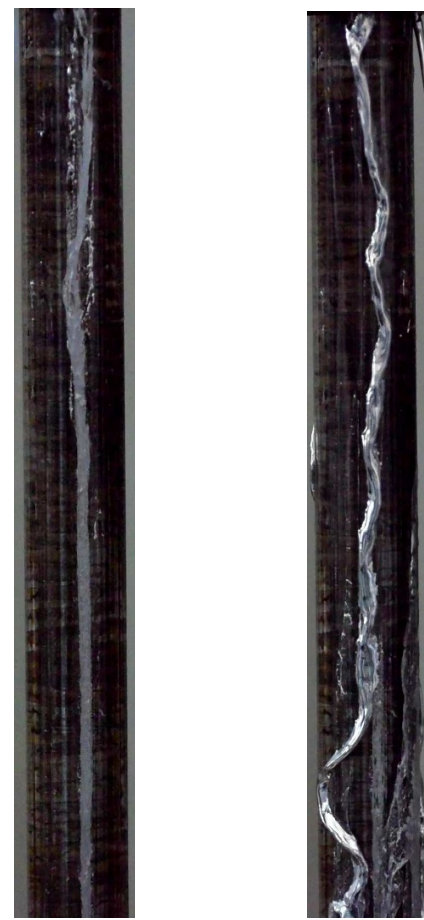

Fig. 4. Examples of liquid metal flow on the quartz cylinder surface.

\section{Conclusions}

The first stage of the experiments on liquid metal motion on the surface of quartz rod was carried out. As a result of the experiments, the characteristics of the melt movement under free flow conditions were determined. The stages of droplet formation were shown. It was found, that the liquid metal prefer to move in the wake region of the first released droplet. The shapes of droplets and its velocity of movement when connected to the rod's surface also were presented. The obtained results are very important to develop models and to understand the processes that take place during the accidents in the core of nuclear power plants.

The work was performed at support of the projects funded by the Russian Science Foundation (RSF № 18-79-10013).

\section{References}

1. M.M. Rahman, Y. Ege, K. Morita et all, Nuclear Engineering and Design, 238, (2008).

2. Md. Soner, Y. Hasegawa, T. Matsumoto et all, Memoirs of the Faculty of Engineering, Kyushu University, 71, 1, (2011).

3. K. Kamiyama , M. Saito , K. Matsuba et all, Journal of Nuclear Science and Technology, 50, 6, (2013).

4. Chatain, D., Lesueur, C., Baland, J.P. Langmuir, 22, 9, (2006). 